\title{
Indirect searches for dark matter with the nEDM spectrometer
}

\author{
S. Roccia ${ }^{1,2}$ and G. Zsigmond ${ }^{3 \star}$ on behalf of the nEDM collaboration at PSI
}

1 Laboratoire de Physique Subatomique et de Cosmologie, Université Grenoble Alpes, CNRS/IN2P3, Grenoble, France

2 Institut Laue-Langevin, CS 20156 F-38042 Grenoble Cedex 9, France

3 Paul Scherrer Institut, 5232 Villigen PSI, Switzerland

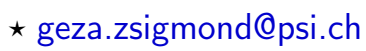

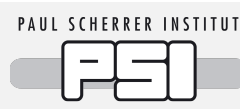

Review of Particle Physics at PSI doi:10.21468/SciPostPhysProc.5

\begin{abstract}
The nEDM apparatus at PSI has been used to search for different dark matter signatures utilizing its high sensitivity to shifts in the neutron precession frequency and its wellcontrolled low magnetic field at the $\mu \mathrm{T}$ level. Such a shift could be interpreted as a consequence of a short-range spin-dependent interaction that could possibly be mediated by axions or axion-like particles, or as an axion-induced oscillating electric dipole moment of the neutron. Another search, based on so-called UCN disappearance measurements, targeted previously reported signals of neutron to mirror-neutron oscillations. These dark matter searches confirmed and improved previous results, as detailed in this review.
\end{abstract}

(c) (1) Copyright S. Roccia, G. Zsigmond et al. This work is licensed under the Creative Commons Attribution 4.0 International License.

Published by the SciPost Foundation.
Received 18-02-2021

Accepted 28-04-2021

Published 06-09-2021

doi:10.21468/SciPostPhysProc.5.028

\subsection{Introduction}

Apart from searching for the electric dipole moment of the neutron, the nEDM apparatus at PSI (Section 27 [1]) is also an excellent tool to search for signatures of dark matter particles. The first potential signature studied was a high precision measurement of the shift in the ratio of the spin-precession frequencies of ultracold neutrons (UCN) and ${ }^{199} \mathrm{Hg}$ atoms. This shift can be interpreted as originated from a possible short-range spin-dependent neutron-nucleon interaction [2]. A second search focused on ultra-low-mass axionlike dark matter. The aforementioned ratio was measured and analyzed as an axion-induced oscillating electric dipole moment of the neutron and an axion-wind spin-precession effect [3]. UCN disappearance experiments were conducted with this apparatus [4] to search for dark matter signatures. It has been proposed starting from the fifties that there could be a mirror copy of the Standard Model (SM) particles, restoring parity conservation in the weak interaction on the global level. Oscillations between a neutral SM particle, such as the neutron, and its mirror counterpart could 
help explain various issues in physics, including dark matter. The neutron electric dipole moment collaboration at PSI conducted an experiment to search for anomalous signals reported before.

Below we summarize the results of our experiments aiming at identifying dark matter signatures with the nEDM apparatus at PSI. A comparison to previous constraints on model parameters will be given.

\subsection{Search for axion-like particles}

The most elegant solution to the strong CP problem is to introduce a global chiral U(1) symmetry, usually named $U(1)_{P Q}$ after the two physicists who first proposed it, R. D. Peccei and H. R. Quinn [5] in 1977. $U(1)_{P Q}$ is spontaneously broken at some energy scale $f_{a}$ producing a pseudo-Nambu-Goldstone boson of the global $U(1)_{P Q}$ symmetry: the axion, as proposed by S. Weinberg and F. Wilczek [6].

One defining feature of the axion is the inverse proportionality of the mass $m_{a}$ to the $U(1)_{P Q}$ symmetry breaking scale $f_{a}$. Therefore, one needs only one parameter to describe the axion's properties. In reference [7], the proportionality factor is computed from first principles and using lattice calculations giving

$$
m_{a}=5.70(6)(4) \mu \mathrm{eV}\left(\frac{10^{12} \mathrm{GeV}}{f_{a}}\right)
$$

The axion is a well-motivated dark matter candidate, as it would solve the strong CP problem and possibly explain the observed abundance (or a fraction) of dark matter. However, observing the axion in the mass range where it would explain the dark matter abundance, requires probing a very weak coupling.

Experimentally, searching for an "axion" outside the window defined by (28.1) is referred to as searching for axionlike particles.

An important feature of the nEDM spectrometer [1] is the mercury co-magnetometer. The search for the neutron electric dipole moment employs the ratio $\mathcal{R}$ of the neutron precession frequency $\left(f_{\mathrm{n}}\right)$ to the mercury one $\left(f_{\mathrm{Hg}}\right)$ using the fact that this ratio is, to first order, free from magnetic field fluctuations. Similarly, this ratio can be used to search for exotic couplings forming a class of experiments called clock comparison. In the following, we focus on how to search for axion-like particles with advanced clock comparison experiments using the ratio $\mathcal{R}$ according to equation:

$$
\mathcal{R} \equiv \frac{f_{\mathrm{n}}}{f_{\mathrm{Hg}}}=\frac{\gamma_{\mathrm{n}}}{\gamma_{\mathrm{Hg}}}\left(1+\frac{\vec{b} \cdot \vec{B}}{B^{2}} \pm\left(d_{\mathrm{n}}-\frac{\gamma_{\mathrm{n}}}{\gamma_{\mathrm{Hg}}} d_{\mathrm{Hg}}\right) \frac{2 E}{h f_{\mathrm{Hg}}}\right)
$$

In this expression $\vec{B}(\vec{E})$ is the applied magnetic (electric) field. These fields are parallel to each other. This ratio is sensitive to an EDM-like coupling (for the neutrons $\left(d_{n}\right)$ or for the mercury atoms $\left(d_{\mathrm{Hg}}\right)$ ) and to any coupling generating a pseudo-magnetic field acting differently on neutrons and mercury atoms so that its effective strength $\vec{b}$ is not null.

Some models predict that low mass axions $\left(m_{a} \leq 0.1 \mathrm{eV} / \mathrm{c}^{2}\right)$ [8] could have been produced in the early universe and would form now a coherently oscillating classical field of amplitude $a$ depending only on the axion's mass $m_{a}$ [9]

$$
a(t)=a_{0} \cos \left(\frac{m_{a} c^{2}}{\hbar} t\right)
$$


The same models predict an oscillation of the ratio $\mathcal{R}$ induced by the coupling [3]

$$
\mathcal{L}=\frac{C_{G}}{f_{a}} \frac{\alpha_{S}}{8 \pi} G_{b \mu \nu} \widetilde{G}^{b \mu \nu}-\frac{C_{N}}{2 f_{a}} \partial_{\mu} a \bar{N} \gamma^{\mu} \gamma^{5} N
$$

where $b$ is the color index while $C_{G}$, the axion-gluon coupling, and $C_{N}$, the axion-nucleon coupling, are model dependent dimensionless parameters. The first term is the axion-gluon coupling. It induces an oscillation of the neutron EDM through the same mechanism as the QCD theta term. The second term is the axion-nucleon coupling. It induces an $E$-independent frequency modulation of the ratio $\mathcal{R}$. In both cases, the frequency of the oscillation depends on the (unknown) mass of the axion, see (28.3). The limits obtained at PSI on the couplings $C_{G}$ and $C_{N}$ for different axion masses are shown in Figure 28.1a and Figure 28.1b respectively.

A limit on a completely different mass range can be obtained searching for a scalar-pseudoscalar coupling between the polarized neutrons and the unpolarized nuclei in the electrodes of the storage chamber. Due to gravity, the neutron density is higher close to the bottom electrode so globally the effective field $\vec{b}$ in (28.2) due to the two electrodes does not cancel out for neutrons (it does for mercury atoms whose density is homogeneous). We searched for the effective field $\vec{b}$ as a shift in the $\mathcal{R}$ ratio correlated with the direction of the applied magnetic field. The obtained limit is shown in Figure 28.1c and compared with other limits established using atoms.

\subsection{Search for mirror neutrons}

Lee and Yang suggested [12] that parity symmetry in the weak interaction could be restored by the existence of a parity conjugated copy of the same set of weakly interacting particles. It was shown later [13] that SM particles would not interact with their mirror counterparts $\left(\mathrm{SM}^{\prime}\right)$ via $\mathrm{SM}$ forces, and $\mathrm{SM}^{\prime}$ would have its own interactions. However there may exist interactions beyond the SM, between neutral SM and $\mathrm{SM}^{\prime}$ particles. The idea that by the introduction of mirror matter, parity and time reversal symmetries could be restored in the weak interactions, and thus in a global sense as well, was detailed further in $[14,15]$. Berezhiani and others proved that the interaction of $\mathrm{SM}$ and $\mathrm{SM}^{\prime}$ particles could answer several open questions in physics: (i) mirror matter could be a viable dark matter candidate [16-21], (ii) it would provide a mechanism to help solve sterile neutrino anomalies [22-24], (iii) SM neutrinos could be endowed with mass [23,25], (iv) it could open up additional channels of CP and baryon number violation, helping to explain baryogenesis and the baryon asymmetry of the universe [26, 27], (v) it could provide a mechanism to relax the Greisen-Zatsepin-Kuzmin (GZK) limit on cosmic rays [28,29]. A comprehensive review can be found in [16,30-32].

In [33] Berezhiani et al. showed that as long as neutrons and their mirror counterparts have the same mass, decay width and gravitational potential, application of a magnetic field, $B$, equal to a mirror magnetic field, $B^{\prime}$, in the same place can induce a degeneracy between the $\mathrm{SM}$ and $\mathrm{SM}^{\prime}$ states, and an $n-n^{\prime}$ oscillation would be possible. The time constant for the coupling, $\tau_{n n^{\prime}}$, could be as low as several seconds. By inducing or destroying the degeneracy, this oscillation could be made visible by means of scanning the applied magnetic field in the experiments.

Pokotilovski proposed [34] that if ultracold neutrons (UCN) would be stored, and they would oscillate into the mirror state, then by such disappearance experiments a signature to mirror states could be probed. First experiments with UCN [35-37] obtained a limit under the assumption of a mirror magnetic field $B^{\prime}=0$ of $\tau_{n n^{\prime}}>448 \mathrm{~s}$ (90\% C.L.) [37]. Reference [38] relaxed the conditions to $B^{\prime} \neq 0$ and set a constraint of $\tau_{n n^{\prime}}>12 \mathrm{~s}$ for $0.4 \mu \mathrm{T}<B^{\prime}<12.5 \mu \mathrm{T}$ (95\% C.L.). In [39], Berezhiani et al. further analysed the above experiments and reported signal-like anomalies for $n-n^{\prime}$ oscillation when $B^{\prime} \neq 0$. Reference [40] again identifies statisti- 


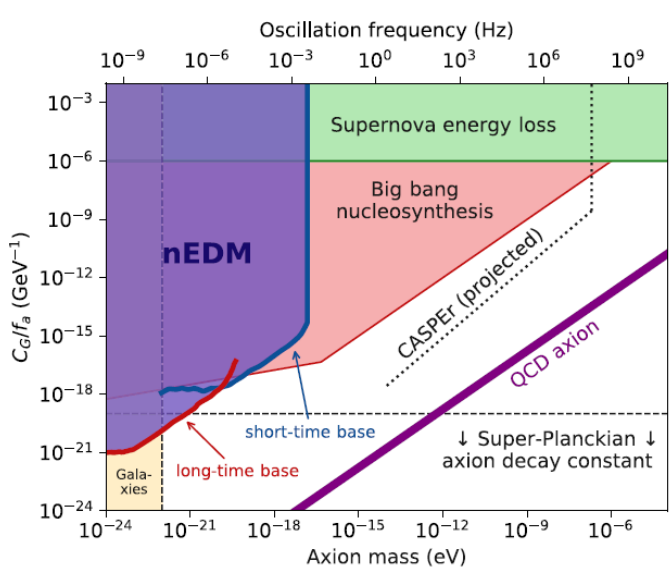

(a)

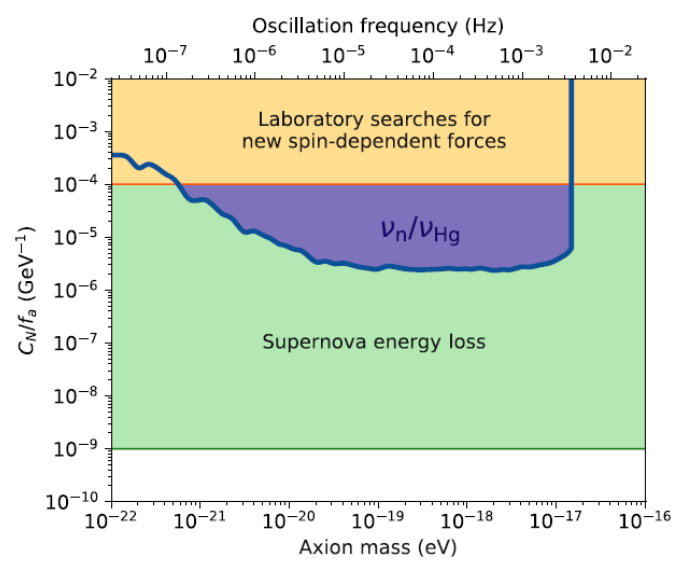

(b)

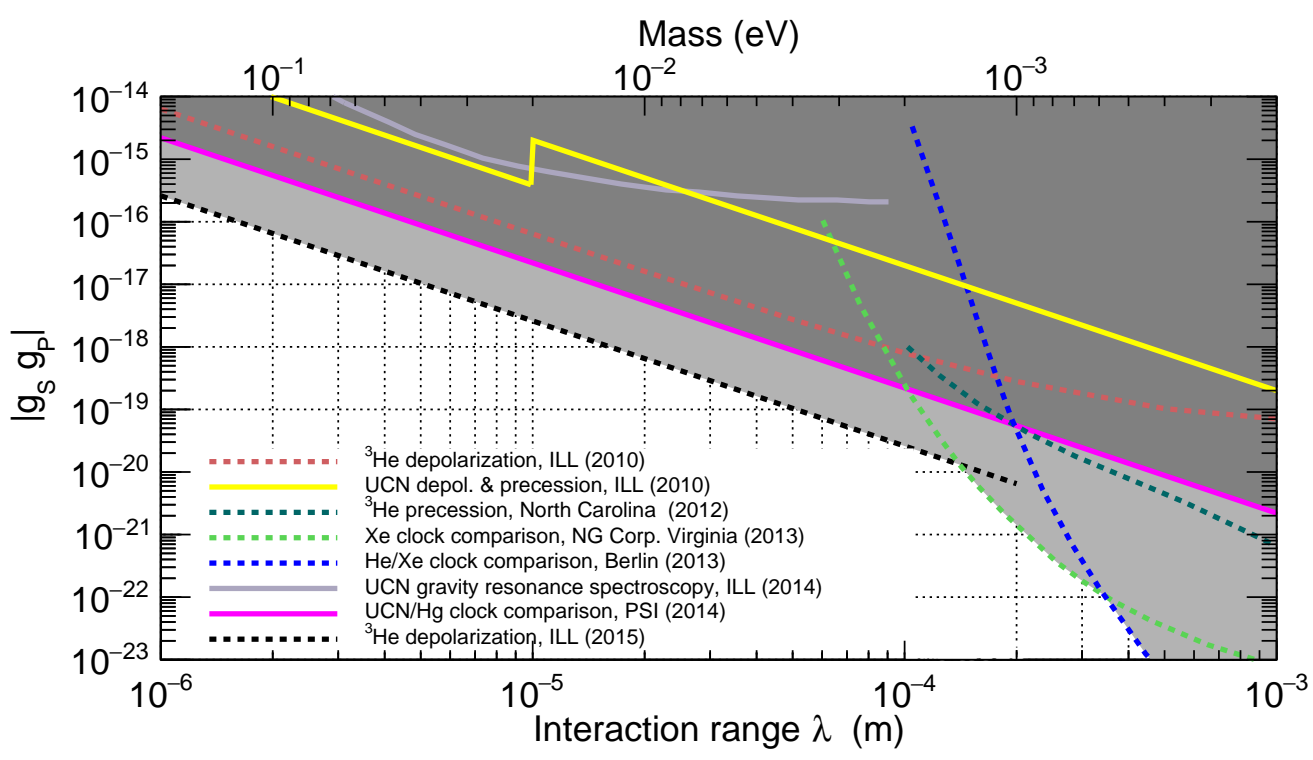

(c)

Figure 28.1: (a) first laboratory-based limit on the axion-gluon coupling $C_{G}$ taken from [3]. (b) limit on the axion-nucleon coupling $C_{N}$. (c) shows the limit established in [10] for the scalar-pseudoscalar coupling for axion or axion-like particle (pink solid line). In this plot, reproduced from [11] (courtesy M. Guigue), the solid lines are constraints established with free neutrons (dark grey excluded zone) while dot-dashed lines are constraints established on bound nucleons (light grey excluded zone).

cally significant signals: a $3 \sigma$ signal from the data in [35], a 5.2 $\sigma$ signal from data in $[36,37]$, and a $2.5 \sigma$ signal in [40].

The potential signals of [39] motivated a new measurement at PSI by the neutron electric dipole moment (nEDM) collaboration [4]. The nEDM apparatus was re-purposed for these storage measurements using unpolarized neutrons in order to increase statistics. The $B$ magnetic field was alternately switched off and on using both polarities, thus modifying the degeneracy between the SM and the assumed SM' energy levels. We did not observe any statistically significant changes in the stored UCN counts from different magnetic field settings, and set constraints on the parameters of $n-n^{\prime}$ oscillation. 
The parameter space of the mirror magnetic field, $B^{\prime}$ and $\left(\tau_{n n^{\prime}} / \sqrt{\cos \beta}\right)$, constrained by different experiments is shown in Figure 28.2 [4]. The angle between the assumed $B^{\prime}$ and the applied magnetic field $B$ is denoted by $\beta$. We also plotted the results from previous searches, including the signal-like anomalies detailed in the caption.

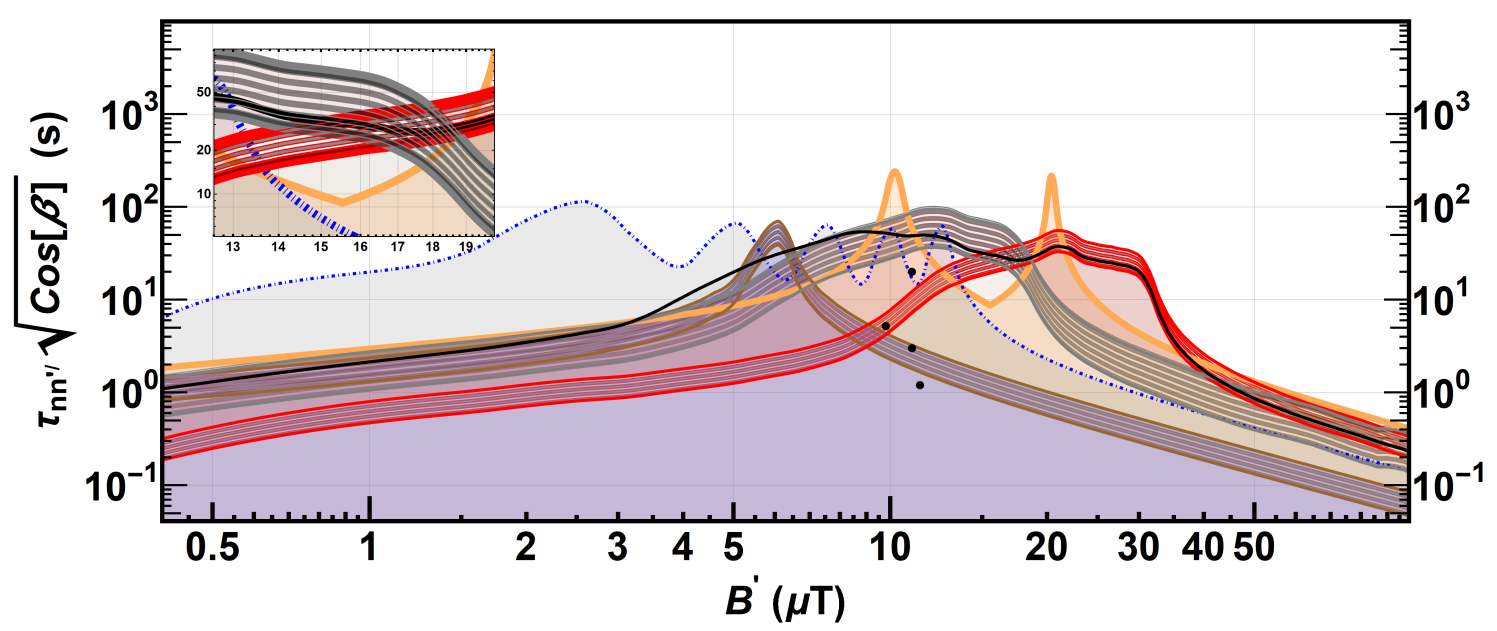

Figure 28.2: Lower limits [4] on the $n-n^{\prime}$ oscillation time, $\tau_{n n^{\prime}}$ at 95\% C.L. while assuming $B^{\prime} \neq 0$. From the asymmetry analysis, the solid orange curve represents the lower limit on $\left(\tau_{n n^{\prime}}^{B^{\prime} \neq 0} / \sqrt{\cos \beta}\right.$. The black curve is the global constraint calculated in [40]. The dot-dashed blue curve represents the lower limit imposed using data in [38] by [40]. The three striped regions are the signals (95\% C.L.): (i) the red striped region, is the signal region calculated in $[39,40]$ from the $5.2 \sigma$ anomaly in [37]; (ii) the brown striped region is the signal calculated in $[39,40]$ from the $3 \sigma$ anomaly in [35]; and (iii) the gray striped region is the signal from the $2.5 \sigma$ anomaly observed in [40]. The black dots indicate the the solution consistent with the statistically significant signals as reported in [39].

As in [33], we considered that the mirror magnetic field $B^{\prime}$ and $\beta$ are constant at the experiment site. The constraints in $[33,35-40]$ were measured at the Institute Laue-Langevin (ILL) in Grenoble. The experiment in [4] was performed at PSI. We worked with the natural assumption that a mirror magnetic field created within the Earth [33] displays approximate rotational symmetry around the Earth's rotation axis, similar to the Earth's magnetic field. Then its components would only change on a level of 5\% between ILL and PSI, and similarly in time, causing a negligible offset on the $B^{\prime}$ axis of Figure 28.2.

The solid orange curve in Figure 28.2 excludes all signal spots (see black dots) reported in [39], for which the experiment at PSI was optimized in 2017. The three signal bands in $[37,39,40]$ exclude each other since they don't overlap at the same mirror magnetic field, $B^{\prime}$. Our analysis excludes three of the five areas where at least two of the signal bands overlap.

\subsection{Outlook}

Competitive searches for dark matter have been conducted using the nEDM spectrometer over the last decade. More ideas are still being explored to extend the limits on axion-like particles: a limit on the oscillating EDM signal at higher frequency (axion mass range up to $10^{-15} \mathrm{eV}$ ) is under study and dedicated data to push the limit of reference [10] are being analyzed.

Additionally, similar searches of axion-like particles are planned with the next generation n2EDM apparatus making use of the much improved sensitivity in the frequency measurements and of the better control over systematic effects. The not yet excluded regions of the signal bands of [40] for mirror neutrons will be a focus of future efforts. 


\section{References}

[1] G. Pignol and P. Schmidt-Wellenburg, The search for the neutron electric dipole moment at PSI, SciPost Phys. Proc. 5, 027 (2021), doi:10.21468/SciPostPhysProc.5.027.

[2] J. Moody and F. Wilczek, New macroscopic forces?, Phys. Rev. D 30, 130 (1984), doi:10.1103/PhysRevD.30.130.

[3] C. Abel et al., Search for axionlike dark matter through nuclear spin precession in electric and magnetic fields, Phys. Rev. X 7, 041034 (2017), doi:10.1103/PhysRevX.7.041034.

[4] C. Abel et al., A search for neutron to mirror-neutron oscillations using the nEDM apparatus at PSI, Phys. Lett. B 812, 135993 (2021), doi:10.1016/j.physletb.2020.135993.

[5] R. Peccei and H. R. Quinn, Constraints imposed by CP conservation in the presence of pseudoparticles, Phys. Rev. D 16, 1791 (1977), doi:10.1103/PhysRevD.16.1791.

[6] F. Wilczek and A. Zee, Instantons and spin forces between massive quarks, Phys. Rev. Lett. 40, 83 (1978), doi:10.1103/PhysRevLett.40.83.

[7] G. Grilli di Cortona, E. Hardy, J. Pardo Vega and G. Villadoro, The QCD axion, precisely, J. High Energy Phys. 01, 034 (2016), doi:10.1007/JHEP01(2016)034.

[8] D. A. Dicus, E. W. Kolb, V. L. Teplitz and R. V. Wagoner, Astrophysical bounds on very low mass axions, Phys. Rev. D 22, 839 (1980), doi:10.1103/PhysRevD.22.839.

[9] J. Preskill, M. B. Wise and F. Wilczek, Cosmology of the invisible axion, Phys. Lett. B 120, 127 (1983), doi:10.1016/0370-2693(83)90637-8.

[10] S. Afach et al., Constraining interactions mediated by axion-like particles with ultracold neutrons, Phys. Lett. B 745, 58 (2015), doi:10.1016/j.physletb.2015.04.024.

[11] M. Guigue, D. Jullien, A. Petukhov and G. Pignol, Constraining short-range spin-dependent forces with polarized ${ }^{3} \mathrm{He}$, Phys. Rev. D 92, 114001 (2015), doi:10.1103/PhysRevD.92.114001.

[12] T. Lee and C.-N. Yang, Question of parity conservation in weak interactions, Phys. Rev. 104, 254 (1956), doi:10.1103/PhysRev.104.254.

[13] I. Kobzarev, L. Okun and I. Pomeranchuk, On the possibility of experimental observation of mirror particles, Sov. J. Nucl. Phys. 3, 837 (1966).

[14] R. Foot, H. Lew and R. Volkas, Possible consequences of parity conservation, Mod. Phys. Lett. A 7, 2567 (1992), doi:10.1142/S0217732392004031.

[15] R. Foot, H. Lew and R. Volkas, A model with fundamental improper space-time symmetries, Phys. Lett. B 272, 67 (1991), doi:10.1016/0370-2693(91)91013-L.

[16] Z. Berezhiani, Mirror world and its cosmological consequences, Int. J. Mod. Phys. A 19, 3775 (2004), doi:10.1142/S0217751X04020075.

[17] H. Hodges, Mirror baryons as the dark matter, Phys. Rev. D 47, 456 (1993), doi:10.1103/PhysRevD.47.456.

[18] Z. Berezhiani, Unified picture of ordinary and dark matter genesis, Eur. Phys. J. ST 163, 271 (2008), doi:10.1140/epjst/e2008-00824-6. 
[19] R. Foot, Mirror dark matter interpretations of the DAMA, CoGeNT and CRESST-II data, Phys. Rev. D 86, 023524 (2012), doi:10.1103/PhysRevD.86.023524.

[20] R. Foot, Mirror dark matter: Cosmology, galaxy structure and direct detection, Int. J. Mod. Phys. A 29, 1430013 (2014), doi:10.1142/S0217751X14300130.

[21] R. Foot, Experimental implications of mirror matter - type dark matter, Int. J. Mod. Phys. A 19, 3807 (2004), doi:10.1142/S0217751X04020087.

[22] V. Berezinsky, M. Narayan and F. Vissani, Mirror model for sterile neutrinos, Nucl. Phys. B 658, 254 (2003), doi:10.1016/S0550-3213(03)00191-3.

[23] E. K. Akhmedov, Z. G. Berezhiani and G. Senjanovic, Planck scale physics and neutrino masses, Phys. Rev. Lett. 69, 3013 (1992), doi:10.1103/PhysRevLett.69.3013.

[24] Z. G. Berezhiani and R. N. Mohapatra, Reconciling present neutrino puzzles: Sterile neutrinos as mirror neutrinos, Phys. Rev. D 52, 6607 (1995), doi:10.1103/PhysRevD.52.6607.

[25] Z. Silagadze, Neutrino mass and the mirror universe, Phys. Atom. Nucl. 60, 272 (1997), arXiv:hep-ph/9503481.

[26] L. Bento and Z. Berezhiani, Leptogenesis via collisions: The Lepton number leaking to the hidden sector, Phys. Rev. Lett. 87, 231304 (2001), doi:10.1103/PhysRevLett.87.231304.

[27] Z. Berezhiani, Neutron-antineutron oscillation and baryonic majoron: low scale spontaneous baryon violation, Eur. Phys. J. C 76, 705 (2016), doi:10.1140/epjc/s10052-0164564-0.

[28] Z. Berezhiani and A. Gazizov, Neutron oscillations to parallel world: Earlier end to the cosmic ray spectrum?, Eur. Phys. J. C 72, 2111 (2012), doi:10.1140/epjc/s10052-0122111-1.

[29] Z. Berezhiani and L. Bento, Fast neutron: Mirror neutron oscillation and ultra high energy cosmic rays, Phys. Lett. B 635, 253 (2006), doi:10.1016/j.physletb.2006.03.008.

[30] Z. Berezhiani, D. Comelli and F. L. Villante, The Early mirror universe: Inflation, baryogenesis, nucleosynthesis and dark matter, Phys. Lett. B 503, 362 (2001), doi:10.1016/S03702693(01)00217-9.

[31] Z. Berezhiani, Through the looking-glass: Alice's adventures in mirror world, In From Fields to Strings: Circumnavigating Theoretical Physics: A Conference in Tribute to Ian Kogan, 2147, doi:10.1142/9789812775344_0055 (2005).

[32] L. Okun, Mirror particles and mirror matter: 50 years of speculations and search, Phys. Usp. 50, 380 (2007), doi:10.1070/PU2007v050n04ABEH006227.

[33] Z. Berezhiani, More about neutron - mirror neutron oscillation, Eur. Phys. J. C 64, 421 (2009), doi:10.1140/epjc/s10052-009-1165-1.

[34] Y. Pokotilovski, On the experimental search for neutron $\rightarrow>$ mirror neutron oscillations, Phys. Lett. B 639, 214 (2006), doi:10.1016/j.physletb.2006.06.005.

[35] G. Ban et al., Direct experimental limit on neutron-mirror-neutron oscillations, Phys. Rev. Lett. 99, 161603 (2007), doi:10.1103/PhysRevLett.99.161603. 
[36] A. Serebrov et al., Experimental search for neutron-mirror neutron oscillations using storage of ultracold neutrons, Phys. Lett. B 663, 181 (2008), doi:10.1016/j.physletb.2008.04.014.

[37] A. Serebrov et al., Preparation of UCN facilities for fundamental research at PNPI, Nucl. Instrum. Meth. A 611, 276 (2009), doi:10.1016/j.nima.2009.07.078.

[38] I. Altarev et al., Neutron to mirror-neutron oscillations in the presence of mirror magnetic fields, Phys. Rev. D 80, 032003 (2009), doi:10.1103/PhysRevD.80.032003, 0905.4208.

[39] Z. Berezhiani and F. Nesti, Magnetic anomaly in UCN trapping: signal for neutron oscillations to parallel world?, Eur. Phys. J. C 72, 1974 (2012), doi:10.1140/epjc/s10052-0121974-5.

[40] Z. Berezhiani, R. Biondi, P. Geltenbort, I. Krasnoshchekova, V. Varlamov, A. Vassiljev and O. Zherebtsov, New experimental limits on neutron - mirror neutron oscillations in the presence of mirror magnetic field, Eur. Phys. J. C 78, 717 (2018), doi:10.1140/epjc/s10052018-6189-y. 